Mittheilung aus dem chemischen Laboratorium der Universität Jena.

\title{
Ueber Tetronsäure;
}

von Ludwig Wolff.

(Eingelaufen am 10. März 1896.)

Die Tetrinsäure gehört, wie ich kürzlich dargelegt habe ${ }^{1}$ ), der Klasse der $\gamma$-Lactone an und verdankt ihren stark ausgeprägten sauren Charakter der Atomgruppirung - $\mathrm{CO}-\mathrm{CH}_{2}-\mathrm{CO}$ oder $-\mathrm{C}(\mathrm{OH})=\mathrm{CH}-\mathrm{CO}$-; für die Säure und ihre Homologen kommen nur mehr die Formeln ${ }^{2}$ )<smiles>CCCCCCC(=O)OC</smiles><smiles>CCOC(=O)C(=O)O</smiles>

in Betracht, zwischen denen eine endgültige Entscheidung vorerst nicht getroffen werden kann.

Die Säuren entstehen ganz allgemein und in guter Ausbeute aus den Monobrommonoalkyl-Acetessigestern, und so durfte man eigentlich erwarten, dass für das Zustandekommen der Reaction vor allem die $\gamma$-Stellung des Bromatomes in den Acetessigestern erforderlich sei. Man sollte vom Bromdimethylacetessigester zur Methyltetrinsäure, vom $\gamma$-Bromacetessigester zu dem einfachsten Gliede, zur Tetronsäure, gelangen können :

$$
\left.\right|_{\mathrm{CH}_{2}-\mathrm{COOO}_{2} \mathrm{H}_{5}} ^{\mathrm{CO}-\mathrm{CH}_{2} \mathrm{Br}}=\left.\right|_{\mathrm{CH}_{3}-\mathrm{CO}} ^{\mathrm{CO}} \mathrm{CH}_{2}^{-\mathrm{O}}+\mathrm{C}_{2} \mathrm{H}_{5} \mathrm{Br}
$$

(Tetronsäure).

Diesbezügliche Versuche sind mit Bromdimethylacetessigester von Moscheles und Cornelius ${ }^{3}$ ) ohne Erfolg ausgeführt

1) Diese Annalen $\mathbf{2 8 8 ,} 1$.

2) Lediglich der Einfachheit lualber bediene ich mich auch in dieser Abhandlung der Ketonformel bei der Besprechung einzelner Reactionen.

3) Ber. d. dentsch. chem. Ges. 21, 2603. 
worden; die Ursache des Misslingens ist zweifellos nur in der geringen Widerstandsfähigkeit zu suchen, welche den $\alpha$-Disubstitutionsproducten der Tetronsäure eigen ist. Ich habe bis jetzt fünf derartige Körper in Händen gehabt, deren Verhalten die vorgetragene Ansicht rechtfertigt; sie bilden das Beobachtungsmaterial für den früher von mir aufgestellten Satz ${ }^{4}$ ).

Es kam mir vor allem darauf an, die Muttersubstanz, die Tetronsäure, kennen $\mathrm{zu}$ lernen. $\mathrm{Zu}$ diesem $\mathrm{Zweck}$ wurde Bromacetessigester sowohl für sich erhitzt als auch mit Kalilösung zersetzt und festgestellt, dass in letzterem Falle die gewünschte Reaction thatsächlich vor sich geht. Freilich gelang es nicht, die Tetronsäure, welche unter den Versuchsbedingungen nicht beständig ist, zu isoliren, aber es wurde ein Condensationsproduct derselben erhalten, das später direct aus Tetronsäure gewonnen werden konnte. Offenbar aus demselben Grunde, das heisst wegen der grossen Veränderlichkeit der Tetronsäure, liess sich letztere auch in dem durch Erhitzen des Esters auf etwa $130^{\circ}$ erhältlichen dickflüssigen Oel nicht auffinden; an ihrer Stelle wurde eine kleine Menge einer krystallinischen Säure von der Zusammensetzung $\mathrm{C}_{4} \mathrm{H}_{3} \mathrm{BrO}_{3}$, d. i. Bromtetronsäure, erhalten und geschlossen, dass dieselbe aus Dibromacetessigester entstanden sei, dessen Bildung sich beim Bromiren des Acetessigesters kaum vermeiden lässt. In der That konnte diese Verbindung, welche sich bei der cingehenden Untersuchung als Bromtetronsäure erwies, in ungleich besserer Ausbeute aus Dibromacetessigester gewonnen werden; mittels nascenten Wasserstoffs liess sie sich leicht in Tetronsäure überführen. Die Umsetzungen finden in folgenden Gleichungen ihren Ausdruck:

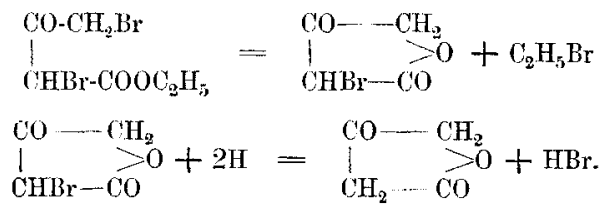

4) Diese Annalen $\mathbf{2 0 8}, 8$ Anm. 
Die Zusammengehörigkeit dieser Säuren und der Tetrinsäure (Methyltetronsäure) erkennt man an einer Reihe durchaus analog verlaufender Reactionen; so an dem Verhalten zu Eisenchlorid, der Beständigkeit der Salze gegen Kohlensäure, der Wirkungsweise des Broms u. s. w.; dagegen verhalten sich die neuen Verbindungen, wie vorauszusehen war, gegen salpetrige Säure anders wie die Methyltetronsäure. Die Tetronsäure ist auch dadurch ausgezeichnet, dass sie sehr leicht gemäss der Gleichung

$$
2 \mathrm{C}_{4} \mathrm{H}_{4} \mathrm{O}_{3}=\mathrm{C}_{3} \mathrm{H}_{4 i} \mathrm{O}_{55}+\mathrm{H}_{2} \mathrm{O}
$$

in die Anhydrotetronsäure übergeht and grosse Neigung hat, Condensationsproducte $\mathrm{zu}$ bilden.

'Tetronsäure giebt mit Brom bei Ausschluss von Feuchtigkeit nur die ursprüngliche Monobromtetronsäure; bei Gegenwart von Wasser ist die Dibromtetronsäure das Endproduct. Letztere wird durch Ammoniak bei gewöhnlicher Temperatur quantitativ in Dibromacetamid und Glycolsäure gespalten; hieraus ergiebt sich die Constitution der drei Säuren:

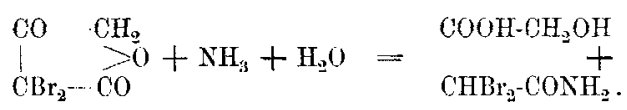

In analoger Weise zersetzt sich, wie früher angegeben wurde, die Nitrosomethyltetronsäure in Oximidopropionsäureamid und Glycolsäure :

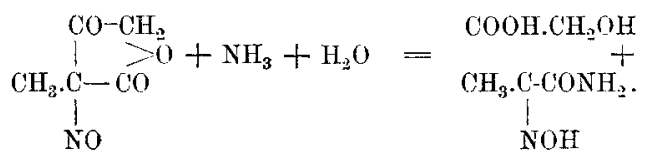

Die Dibromtetronsüure ist wenig beständig und zeigt ein auffallendes Verhalten; sie verwandelt sich auch bei niedriger Temperatur spontan in Tribrommethylketol und Bromtetronsäure, es findet also eine Uebertragung eines Bromatomes von einem Molekül anf das andere statt:

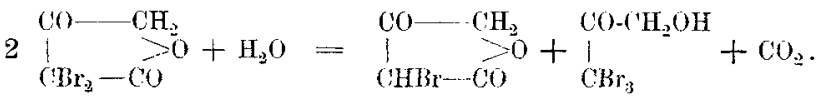


Quantitativ vollzieht sich die Aufspaltung zu dem Ketol, wenn die Säure mit überschüssigem Brom einige Zeit in Berührung bleibt:

$$
\underset{\mathrm{CBr}_{2}-\mathrm{CO}}{\mathrm{CO}-\mathrm{CH}_{\mathrm{O}}}+2 \mathrm{Br}+\mathrm{H}_{2} \mathrm{O}={\underset{\mathrm{CBr}}{3}}_{\mathrm{CO}-\mathrm{CH}_{2} \mathrm{OH}}^{1}+\mathrm{CO}_{2}+\mathrm{Br} \cdot \mathrm{H} \text {. }
$$

Das Tribromketol wird durch Sodalösung in Bromoform und Glycolsäure zerlegt.

Tetronsäure und Bromtetronsäure werden durch salpetrige Säure in Oximidotetronsäure übergeführt, und diese giebt mit salzsaurem Hydroxylamin in kalter wässriger Lösung momentan Dioximidobutyrolacton :

$$
\begin{aligned}
& \left.\right|_{\mathrm{CH}_{2}-\mathrm{CO}_{\mathrm{CO}}^{\mathrm{CO}}-\mathrm{CH}_{3}} ^{\mathrm{CHNO}_{2}}=\left.\right|_{\mathrm{CNOH}-\mathrm{CO}} ^{\mathrm{CO}-\mathrm{CH}_{2}}+\mathrm{H}_{2} \mathrm{O}
\end{aligned}
$$

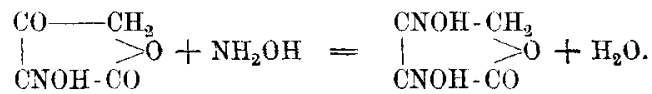

Analog verhält sich die Brommethyltetronsäure gegen salzsaures Hydroxylamin:

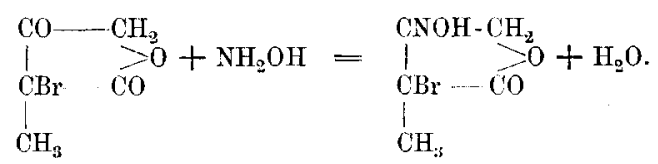

Diese Umsetzung (mit salzsaurem Hydroxylamin) bietet an sich nichts Neues und konnte vorausgesehen werden; sie hat hier aber ein besonderes Interesse, weil die Tetronsäure, die Bromtetronsäure und Methyltetronsäure unter den gleichen Bedingungen mit salzsaurem Hydroxylamin nicht in die entsprechenden Oximidokörper übergehen. In der Wärme wirken freies und auch salzsaures Hydroxylamin ein, allein die dabei in kleiner Menge auftretenden krystallinischen Verbindungen verdanken ihre Entstehung einer weitergehenden Zersetzung ${ }^{5}$ ).

5) Die ans Methyltetronsäure $\left(\mathrm{C}_{5} \mathrm{H}_{6} \mathrm{O}_{3}\right)$ erhaltene Verbindung schmolz bei $166^{\circ}$ unter Zersetzung und gab bej der Analyse folgende, für die Formel $\mathrm{C}_{4} \mathrm{H}_{8} \mathrm{~N}_{2} \mathrm{O}_{3}$ gut stimmende Werthe: C 36,$36 ; \mathrm{H} 5,89$; N 20,98. Tch betrachte indessen die Zusammensetzung des Körpers nicht für endgültig festgestellt. 
Die schon in der ersten Abhandlung discutirte Frage, ob die freien Säuren sich von der Keton- oder Hydroxylformel<smiles>O=C(O)C1CCOCC1=O</smiles>

( $\mathrm{R}=$ Alkyl, $\mathrm{Br}$ oder $\mathrm{H}$ ) ableiten, muss auch heute noch als ungelöst angesehen werden. Wenn meine mit der Tetrinsäure angestellten Versuche fast durchweg zu Gunsten der Ketonformel sprechen, und auch das Verhalten der Tetronsäure und Bromtetronsäure zu salpetriger Säure mit dieser Formel in Einklang steht, so lassen andere Beobachtungen doch schwerwiegende Bedenken gegen diese Auffassung aufkommen. Es muss besonders darauf hingewiesen werden, dass die $\alpha$-mono- und $\boldsymbol{c}$-disubstituirten Tetronsäuren sich gegen salzsaures Hydroxylamin durchaus verschieden verhalten. Die Disubstitutionsproducte, welche sich von der Ketonformel ableiten müssen, werden momentan in Oximidolactone verwandelt, die Monoderivate gaben derartige Verbindungen nicht. Ein solcher Unterschied im Verhalten weist auf verschiedenartige Constitution hin.

Zu einem ähnlichen Resultate bezüglich der Constitution der Salze gelangt man bei Berücksichtigung der relativ grossen Beständigkeit der Bromtetronsäure in alkalischer Lösung; die Säure entspricht in dieser Beziehung den ungesättigten halogenirten Säuren. Die gesättigten halogenirten Fettsäuren dagegen und namentlich die Ketonsäuren geben das Halogen so leicht ab, dass in den meisten Fällen schon bei der Salzbildung eine Zersetzung eintritt, sie zeigen das Verhalten der leicht veränderlichen $\alpha$-Brommethyl- und $\alpha$-Dibromtetronsäure. Es darf hier auch die Thatsache hervorgehoben werden, dass bromtetronsaures Silber sich unzersetzt aus kochendem Wasser umkrystallisiren lässt, eine gewiss auffällige Erscheinung, wollte man annehmen, dass das Brom- und das Silberatom ein und demselben Kohlenstoffatom angehören. Zwar ist, wie ich gefunden habe, das Silbersalz des Bromdinitromethans 


$$
\mathrm{C}_{\mathrm{Ag}}^{-\left(\mathrm{NO}_{2}\right)_{3}}
$$

in heisser wässriger Lösung recht beständig, allein die Constitution dieser oder einiger anderer in Betracht zu ziehender Salze ist noch nicht mit aller Sicherheit festgestellt.

Dass den Benzoylverbindungen (Tetronsäure, Methyltetronsäure und Benzyltetronsäure) und den Estern (Methyl-, Aethylund Benzyltetronsäure) die Hydroxylformel zu Grunde liegt, habe ich früher bereits erwähnt; sie lassen sich, wie Moscheles und Cornelius ${ }^{6}$ ) zuerst gefunden haben, sebr leicht in die zugehörigen Tetronsäuren spalten. Letztere Thatsache genügt Freer ${ }^{7}$ ), um die Hydroxylformel für die freien Säuren als bewiesen ansehen zu können - mit demselben Rechte, mit dem er das Aceton als ungesättigten Alkohol betrachtet.

\section{Experimenteller Theil.}

Von Ludwig Wolff und Carl Schwale.

\section{Darstellung der Bromtetronsäure.}

Acetessigester wird in Portionen von $100 \mathrm{~g}$ mit dem gleichen Volumen Aether verdünnt und in der gewöhnlichen Weise mit $246 \mathrm{~g}$ Brom versetzt. Zur Entfernung der Bromwasserstoffsäure schüttelt man die Lösung mit viel kaltem Wasser wiederholt durch, trennt die Schichten, entwässert sorgfältig mit Chlorcalcium und treibt den Aether auf dem Wasserbade ab. Je $500 \mathrm{~g}$ des zurückbleibenden Dibromacetessigesters erhitzt man dann unter vermindertem Drucke $(30-40 \mathrm{~mm})$ in einem starkwandigen Rundkolben zwei bis drei Stunden im Oelbade auf $120-130^{\circ}$, saugt die abgeschiedene Krystallmasse möglichst scharf ab, wäscht mit Benzol gut aus und wiederholt die Operation mit der Mutterlauge noch zwei bis lrei Mal. Das beim

6) Ber. d. deutsch. chem Ges. $\mathbf{2 1}, 2603$.

7) Amer. chem. Journ. 17, 779. 
erstmaligen Erhitzen entstehende Product ist gelb gefärbt nud ziemlich rein; die später ausfallenden Portionen sind braun und schmierig und werden zweckmässig für sich gereinigt.

$500 \mathrm{~g}$ Dibromacetessigester liefern $110-115 \mathrm{~g}$ robe Säure ${ }^{8}$ ). Zur Reinigung krystallisirt man sie aus kochendem Wasser (mit Thierkohle) um und erhält sie so in Form feiner, hellgelber Nädelchen, die für die meisten Umsetzungen rein genug sind.

Die ganz reine Säure bildet vollkommen weisse Nädelchen oder Prismen, welche sich bei $160^{\circ}$ gelb färben und bei $183^{0}$ unter vollständiger Zersetzung schmelzen. Sie löst sich leicht in Alkohol und kochendem Wasser, weniger leicht in Aether und kaltem Wasser, schwer in Ligroïn, Chloroform und Schwefelkohlenstofi.

I. $0,2011 \mathrm{~g}$ gaben $0,1954 \mathrm{CO}_{2}$ und $0,\left(0301 \mathrm{H}_{2} \mathrm{O}\right.$.

II. 0,2246 " $" 0,2212 \mathrm{CO}_{2} \quad " \quad 0,0337 \mathrm{H}_{2} \mathrm{O}$.

III. 0,1864 $\mathrm{g} \quad, \quad 0,1956 \mathrm{AgBr}$.

IV. $0,3519 \mathrm{~g}, 0,0,3710 \mathrm{AgBr}$.

\begin{tabular}{cccccc} 
& Bercechnet für & \multicolumn{4}{c}{ diefunden } \\
\cline { 3 - 7 } & $\mathrm{C}_{4} \mathrm{H}_{3} \mathrm{BrO}_{3}$ & I. & II. & III. & IV. \\
$\mathrm{C}$ & 26,81 & 26,50 & 26,86 & - & - \\
$\mathrm{H}$ & 1,67 & 1,66 & 1,67 & - & - \\
$\mathrm{Br}$ & 44,69 & - & - & 44,63 & 44,86
\end{tabular}

Molekulargewichtshestimmung nach Beckmann (Siedemethode).

$0,8912 \mathrm{~g}$ in $18,59 \mathrm{~g}$ Alkohol ergaben eine Siedepunktserhöhung voul $0,326^{\circ}$.

Berechnet für $\mathrm{C}_{4} \mathrm{H}_{3} \mathrm{BrO}_{3}$

il,

Eine weitere Bestimmung nach Raoult's Verfahren in Eisessig ergab den Werth 173. Es kann demnach einem Zweifel nicht mehr unterliegen, dass der Bromtetronsäure und somit

8) Die Ausbente lässt sehr zu wünschen übrig, wenn das Erhitzen unter Atmosphärendruck vorgenommen wird; offenbar entstehen Nebenproducte, welche zersetzend auf den Ester oder die Bromtetronsäure einzuwirken vermögren und unter vermindertem Irucke überdestilliren. 
auch der Tetronsäure die einfachen Formeln mit vier Kohlenstoffatomen zukommen $^{9}$ ).

In der wässrigen Lösung der Bromtetronsäure ruft Eisenchlorid eine dunkelrothe Färbung hervor, welche auf Zusatz von Salzsäure verschwindet. Von Natriumnitritlösung wird die Säure mit violetter Farbe aufgenommen, und letztere schlägt bei Zusatz von etwas Natronlauge oder Salzsäure in gelb um.

Die Säure reducirt $F$ ehling'sche Lösung und Permanganat. Gegen kochendes Wasser, Ammoniak oder Sodalösung ist sie recht beständig; aber beim Erhitzen mit Wasser anf $120^{\circ}$ oder bei längerem Kochen mit viel Natronlauge wird sie vollkommen zersetzt. Sie treibt aus den Carbonaten Kohlensäure mit Leichtigkeit aus und wird aus der Lösung ihrer Salze durch Einleiten von Kohlensäure nicht in Freiheit gesetzt.

Das Calcium - und Baryumsalz sind in Wasser leicht löslich und krystallisiren in weissen Nädelchen oder Prismen.

Das Silbersalz, $\mathrm{C}_{4} \mathrm{H}_{2} \mathrm{BrO}_{3} \mathrm{Ag}$, wird aus den Lösungen der Erdalkalisalze durch salpetersaures Silber als dicker, weisser Niederschlag gefällt und lässt sich aus kochendem Wasser ohne Zersetzung umkrystallisiren. Es bildet verfilzte Nadeln, welche beim Erhitzen verpuffen.

$0,2081 \mathrm{~g}$ gaben $0,1263 \mathrm{CO}_{3}$ und $0,0149 \mathrm{H}_{2} \mathrm{O}$.

$0,2480 \mathrm{~g} \quad " \quad 0,1630 \mathrm{AgBr}$.

$\begin{array}{lcr} & \text { Berechnet für } & \text { Gefiuden } \\ & \mathrm{C}_{4} \mathrm{H}_{2} \mathrm{BrO}_{3} \mathrm{Ag} & \\ \mathrm{C} & 16,78 & 16,55 \\ \mathrm{H} & 0,70 & 0,79 \\ \mathrm{Ag} & 37,76 & 37,74\end{array}$

9) Durch die Untersuchungen von Moscheles und Cornelius und namentlich von Walden (Ber. d. dentsch. chem. ('es. 24, 2025) ist die einfache Formel $\mathrm{C}_{5} \mathrm{H}_{6} \mathrm{O}_{2}$ für die Tetrinsänre ausser allen Zweifel gestellt; die kürzlich von Freer und Miller (Americ. chem. Journ. 17, 779) veröffentlichten Molekulargewichtsbestimmungen fuihren, wie nicht anders erwartet werden kounte, zu dem gleichen Resultate. 
Bei der Zersetzung des Dibromacetessigesters entsteht neben Bromäthyl, das wir in reinem Zustande isolirt haben, ein Gemisch öliger, mit Wasserdämpfen leicht flüchtiger Verbindungen, welche die Augen stark angreifen. Bei der Destillation ging der grösste Theil von $140-150^{\circ}$ über und gab mit Bisulfitlösung eine krystallinische Doppelverbindung. Offenbar liegen gebromte Ketone vor, deren Bildung für den Reactionsverlauf von untergeordneter Bedeutung ist.

\section{loarstellung der Tetronsïure.}

Bromtetronsäure wird in Portionen von $30 \mathrm{~g}$ in Sodalösung aufgenommen und mit $400 \mathrm{~g}$ vierprocentigem Natriumamalgam in Antheilen von je $50 \mathrm{~g}$ versetzt; während der Operation leitet man durch die Flüssigkeit, die möglichst concentrirt und kalt gehalten wird, einen Kohlensäurestrom, säuert nach beendigter Umsetzung (nach vier bis fünf Stunden) mit Schwefelsäure $\left(1: 1 \mathrm{H}_{2} 0\right)$ stark an und schüttelt die Lösung alsbald 20 bis 25 Mal mit Aether aus. Beim Verdunsten des Aethers hinterbleibt die Tetronsäure in Form wassêrheller Tafeln, welche mit etwas Aether nachgewaschen werden und dann für die meisten Reactionen rein genug sind. Die Ausbeute ist gut.

Aus einer warmen Mischung von Alkohol und Ligroin krystallisirt die Süure in glänzenden Tafeln, aus lauwarmem Wasser in flächenreichen, glasglänzenden Prismen. Sie sintert bei $135^{\circ}$ und schmilzt bei $141^{\circ}$.

I. $0,2007 \mathrm{~g}$ gaben $0,3510 \mathrm{CO}_{2}$ und $0,0721 \mathrm{H}_{2} \mathrm{O}$.

II. $0,1997 \mathrm{~g} \quad, \quad 0,3503 \mathrm{CO}_{\mathrm{g}} \quad, \quad 0,0715 \mathrm{H}_{2} \mathrm{O}$.

III. $0,2001 \mathrm{~g} \quad, \quad 0,3526 \quad \mathrm{CO}_{2} \quad, \quad 0,0678 \mathrm{H}_{2} \mathrm{O}$.

\begin{tabular}{ccrrr} 
& Berechnet für & \multicolumn{3}{c}{ Gefunden } \\
\cline { 3 - 5 } & $\mathrm{C}_{4} \mathrm{H}_{4} \mathrm{O}_{3}$ & I. & II. & III. \\
$\mathrm{C}$ & 48,00 & 47,69 & 47,84 & 48,05 \\
$\mathrm{H}$ & 4,00 & 3,99 & 3,98 & 3,76
\end{tabular}

Die Tetronsäure löst sich leicht in warmem Alkohol und Wasser, ziemlich schwer in Aether, Benzol, Chloroform und Ligroïn. Sie ist eine sehr starke, einbasische Säure, welche mit Leichtigkeit Bicarbonate zersetzt und aus ibren Salzlösungen 
durch Kohlensäure nicht in Freiheit gesetzt wird. Eisenchlorid erzeugt in der wässrigen Lösung eine dunkelrothe, Natriumnitrit eine violette Färbung; auch gegen Fehling'sche Lösung und Permanganat verhält sie sich wie Bromtetronsäure.

Es ist bereits erwähnt worden, dass die Tetronsäure, wie auch ihre Salze, in wässriger Lösung sich leicht in Anhydrotetronsäure verwandelt.

Tetronsaures Calcium, $\left(\mathrm{C}_{4} \mathrm{H}_{3} \mathrm{O}_{3}\right)_{2} \mathrm{Ca}+2^{1} /{ }_{2} \mathrm{H}_{2} \mathrm{O}$, wurde gewonnen durch Absättigen der Säure mit Calciumcarbonat in der Kälte und Eindunsten der Lösung !im Vacuum. Es löst sich leicht in Wasser und krystallisirt daraus in Nadeln, welche vier Moleküle Wasser $\mathrm{zu}$ enthalten scheinen, von denen $1_{1 / 2}^{1 / 2}$ Moleküle sehr langsam beim Liegen des Salzes an der Luft entweichen. Das Salz zeigte erst nach vierwöchentlichem Stehen an der Luft Gewichtsconstanz und hatte dann noch $2^{1 / 2}$ Moleküle Wasser.

$0,2239 \mathrm{~g}$, lufttrocken, verloren über Schwefelsä̈ure nnd bei $170^{\circ}$ $0,0355 \mathrm{~g}$ und gaben $0,1064 \mathrm{CasO}_{4}$.

$\begin{array}{ccc}\text { Berechnet fïr } & \text { Gefinden } \\ \left(\mathrm{C}_{4} \mathrm{H}_{3} \mathrm{O}_{3}\right)_{2} \mathrm{Ca}+2{ }^{1} /{ }_{2} \mathrm{H}_{2} \mathrm{O} & \\ \mathrm{Ca} & 14,13 & 14,16 \\ \mathrm{H}_{2} \mathrm{O} & 15,90 & 15,86\end{array}$

In der wässrigen Lösung erzeugt essigsaures Kupfer eine grüne Färbung; nach einiger Zeit scheiden sich grüne Nädelchen des Kupfersalzes aus.

Das Silbersalz ist in Wasser leicht löslich.

Bei unseren ersten Versuchen zur Darstellung der Tetronsäure liessen wir das Natriumamalgam in der gewöhnlichen Weise, ohne Kohlensäure durch die Flüssigkeit zu leiten, einwirken und erhielten neben Tetronsäure und Anhydrotetronsäure eine schön krystallisirende, bromfreie Säure, welche zufolge der Analyse nach der Formel $\mathrm{C}_{10} \mathrm{H}_{10} \mathrm{O}_{6}$ zusammengesetzt ist.

I. $0,2056 \mathrm{~g}$ gaben $0,4003 \mathrm{CO}_{2}$ und $0,0810 \mathrm{H}_{2} \mathrm{O}$.

II. $0,2289 \mathrm{~g} \quad, \quad 0,4450 \mathrm{CO}, \quad, \quad 0,0875 \mathrm{H}_{2} \mathrm{O}$. 


\begin{tabular}{ccrr} 
& Berechnet für & \multicolumn{2}{c}{ Gefunden } \\
C & $\mathrm{C}_{10} \mathrm{H}_{10} O_{6}$ & \multicolumn{1}{c}{} & I. \\
$\mathrm{H}$ & 53,09 & 53,09 & 53,03 \\
$\mathrm{H}$ & 4,42 & 4,37 & 4,24
\end{tabular}

Die Säure krystallisirt aus heissem Wasser in spitzen, glasglänzenden Nadeln oder Prismen, die bei $209^{\circ}$ unter Gasentwicklung schmelzen. Sie löst sich sehr leicht in Alkohol, ziemlich leicht in Chloroform, schwer in kaltem Wasser, Aether und Ligroïn und giebt noch die Eisenchlorid- und Natriumnitritreaction der Tetronsäure.

Die Verbindung wurde bis jetzt nicht eingehend untersucht; sie könnte entstanden sein durch Condensation von Tetrònsäure mit Acetaldehyd nach der Gleichung:

$$
2 \mathrm{C}_{4} \mathrm{H}_{4} \mathrm{O}_{3}+\mathrm{C}_{2} \mathrm{H}_{4} \mathrm{O}=\mathrm{C}_{10} \mathrm{H}_{10} \mathrm{O}_{6}+\mathrm{H}_{2} \mathrm{O} \text {. }
$$

Phenylhydrazid oder -hydrazon der Tetronsäure,

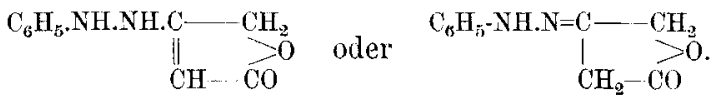

Wenv man Tetronsäure, in Wasser gelöst, mit einer alkoholischen Lösung der berechneten Menge Phenylhydrazin mischt, so scheidet sich nach einiger Zeit ein Oel ab, das bald krystallinisch erstarrt. Aus verdünntem Alkohol krystallisirt die Verbindung in weissen Prismen, die bei $128^{\circ}$ schmelzen. $0,2004 \mathrm{~g}$ gaben $0,4648 \mathrm{CO}_{2}$ und $0,0947 \mathrm{H}_{2} \mathrm{O}$.

$0,2008 \mathrm{~g} \quad, 25,2 \mathrm{ccm}$ Stickgas bei $15^{0}$ und $756 \mathrm{~mm}$ Druck.

$\begin{array}{cr}\text { Berechnet für } & \text { Gefunde } \\ \mathrm{C}_{10} \mathrm{H}_{10} \mathrm{~N}_{\mathbf{2}} \mathrm{O}_{2} & \\ 63,16 & 63,25 \\ 5,26 & 5,25 \\ 14,74 & 14,62\end{array}$

Leicht loslich in warmem Alkohol und Chloroform, schwer löslich in Aether, Benzol und Wasser. Concentrirte Schwefelsäure löst die Verbindung mit blauvioletter Farbe auf, die auf Zusatz von etwas Kaliumbichromat in roth umschlägt.

Der Körper hat keine sauren Eigenschaften mehr, wird aber ron Soda sehr langsam, etwas rascher von Natronlauge, 
mit gelber Farbe gelöst. Concentrirte Salzsäure spaltet ihn in seine Componenten.

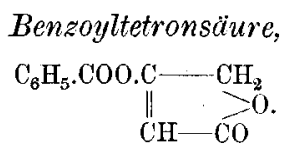

Diese Verbindung scheidet sich krystallinisch ab, wenn man die wässrige Lösung der Tetronsäure mit Benzoylchlorid unter zeitweiligem Zusatz von Natriumcarbonat schüttelt. Die auf Thon getrockneten Krystalle wurden aus einer warmen Mischung von Chloroform und Ligroin umkrystallisirt und in Form von Blättchen oder glänzenden Prismen erhalten, deren Schmelzpunkt bei $120^{\circ}$ liegt.

$0,1760 \mathrm{~g}$ gaben $0,4166 \mathrm{CO}_{2}$ und $0,0615 \mathrm{H}_{2} \mathrm{O}$.

$\begin{array}{lcr} & \text { Berechnet fiür } & \text { Gefunden } \\ & \mathrm{C}_{11} \mathrm{H}_{8} \mathrm{O}_{4} & \\ \mathrm{C} & 64,70 & 64,56 \\ \mathrm{H} & 3,92 & 3,88\end{array}$

Ziemlich leicht löslich in Chloroform, Alkohol und Benzol, weniger leicht in Aether, schwer in Ligroïn. Von Wasser und kalter Sodalösung wird die Verbindung kaum aufgenommen, heisse Sodalösung zerlegt sie leicht in Tetronsäure und Benzoësäure ${ }^{10}$ ).

Einwirkung von Brom auf' Tetronsïlure.

Dibromtetronsëure,<smiles>C1O[C@H]2CO[C@H]1C2</smiles>

Tetronsäure, welche in Chloroform suspendirt ist, verbindet sich leicht mit Brom, und die rothe Farbe bleibt erhalten, wenn auf ein Molekül Säure zwei Atome Brom in Reaction

10) In analoger Weise lässt sich die kürzlich von Freer und Miller (loc. cit.) beschriebene Benzoyltetrinsäure, $\mathrm{C}_{12} \mathrm{H}_{30} \mathrm{O}_{4}$, darstellen; sie krystallisirt aus einem Gemisch ron Chloroform und Iigroin in Form von Nädelchen, welche bei $132^{\circ}$ schmelzen und von warmer Sodalösung in Tetriusäure zerlegt werden. 
getreten sind. Das so entstandene Product schmilat bei $182^{\circ}$ und erweist sich als identisch mit der ursprünglichen Monobromtetronsäure.

$0,2304 \mathrm{~g}$ gaben $0,2411 \mathrm{AgBr}$.

$\begin{array}{ccc} & \text { Berechnet für } & \text { Gefunden } \\ \mathrm{Br} & \mathrm{C}_{4} \mathrm{H}_{3} \mathrm{BrO}_{3} & \\ \mathbf{4 4 , 6 9} & \mathbf{4 4 , 5 3}\end{array}$

Führt man aber den Versuch bei Gegenwart von etwas Wasser aus, so vermag die in Chloroform suspendirte Säure beim Schütteln mit Brom genau vier Atome des letzteren aufzunehmen; es entsteht glatt die Dibromtetronsäure. Zu ihrer Darstellung geht man natürlich von der Bromtetronsäure aus, die in fein zerriebenem Zustande in dem doppelten Gewichte eiskalten Wassers suspendirt und dann mit der berechneten Menge Brom (zwei Atome) bis zur bleibenden hellrothen Farbe versetzt wird. Beim Schütteln bekommt man eine klare Lösung, welche Bromwasserstoff enthält, beim Erwärmen lebhaft Kohlensäure entwickelt und mit Sodalösung Bromoform (Siedep. $146^{\circ}$; Schmelzp. $9^{0}$ ) ausscheidet. Man schüttelt die Flüssigkeit zweimal mit Aether aus und verdunstet letzteren im Vacuum, nachdem er bei niedriger Temperatur mit schwefelsaurem Natrium entwässert worden ist. Es hinterbleibt zunächst ein Oel, das bald zu weissen, in Wasser und Aether leicht löslichen Tafeln erstarrt und nach Bildungsweise und Verhalten das Dibromsubstitutionsproduct der Tetronsäure ist; wir haben dasselbe in analysenreinem Zustande nicht erhalten können. Bei $0^{\circ}$ und Abschluss von Feuchtigkeit hält sich die Verbindung einige Zeit anscheinend unverändert, aber schon nach Ablauf eines Tages wird die Zersetzung daran kenntlich, dass das Product in Wasser oder Aether nicht mehr völlig löslich ist. An der Luft bei Zimmertemperatur verwandeln sich die Krystalle unter Abgabe von Kohlensäure bald in eine schmierige, in Wasser wenig lösliche Masse, welche allmählich wieder krystallinische Structur annimmt und dann aus einem Gemisch von Tribrommethylketol, rückgebildeter Bromtetron- 
säure und vermuthlich Dibrommethylketol (Schmelzp. 90-92 ${ }^{\circ}$ ) besteht. Das mit Wasser gewaschene, kleinkrystallinische Product schmilzt unter Gasentwicklung bei etwa $90^{\circ}$ und lässt sich durch Umkrystallisiren aus heissem Aceton oder Methylalkohol in reines Tribrommethylketol überführen.

$0,2066 \mathrm{~g}$, über Schwefelsäure getrocknet, gaben $0,0874 \mathrm{CO}_{2}$ und $0,0202 \mathrm{H}_{2} \mathrm{O}$.

$0,1965 \mathrm{~g}$, über Schwefelsäture getrocknet, gaben $0,3569 \mathrm{AgBr}$.

$\begin{array}{lcr} & \text { Berechnet für } & \text { Gefunde } \\ & \mathrm{C}_{3} \mathrm{H}_{3} \mathrm{Br}_{3} \mathrm{O}_{2} & \\ \mathrm{C} & 11,57 & 11,53 \\ \mathrm{H} & 0,96 & 1,08 \\ \mathrm{Br} & 77,17 & 77,29\end{array}$

In der Mutterlange dieser Verbindung kann Bromtetronsäure leicht nachgewiesen werden.

Diese Umsetzung der Dibromtetronsäure in Bromtetronsäure und Tribrommethylketol ist so merkwürdig, dass wir zuerst annahmen, diese Producte seien der Dibromtetronsäure von Anfang an beigemengt gewesen; es konnte einerseits etwas Bromtetronsäure der Bromirung entgangen, andererseits die Dibromtetronsäure unter dem Einfluss des Broms in das Ketol verwandelt worden sein. Eine solche Annahme ist indessen nicht berechtigt auf Grund der folgenden Versuche.

1) Es wurden $5 \mathrm{~g}$ Bromtetronsäure, in $30 \mathrm{~g}$ Eiswasser suspendirt, mit $5 \mathrm{~g}$ Brom (theoretisch 4,4) versetzt; die Lösung wurde nach einstündigem Stehen, - sie enthielt noch freies Brom, - mit Aether extrahirt und letzterer im Vacuum verdunstet. Zurück blieb ein Oel, dessen frisch bereitete, kalte wässrige Lösung weder mit Eisenchlorid noch mit Natriumnitrit die für Bromtetronsäure charakteristische Färbung gab; nach halbstündigem Stehen der Lösung bei Zimmertemperatur liess sich Bromtetronsäure durch die rothe Färbung, welche die genannten Reagentien in der Flüssigkeit verursachten, erkennen.

Das Oel blieb dann drei Tage sich selbst überlassen, wobei es sich in eine Krystallmasse verwandelte, aus der reine Bromtetronsäure gewonnen wurde. 
2) $5 \mathrm{~g}$ Bromtetronsäure wurden in Eiswasser mit nur $3,5 \mathrm{~g}$ Brom tüchtig geschüttelt; die filtrirte, farblose Lösung gab an Aether ein Oel ab, das nach dreitägigem Stehen krystallinisch wurde und beim Umkrystallisiren aus Aceton reines Tribrommethylketol lieferte.

Dibromtetronsäure wird durch kohlensaure Alkalien in gleicher Weise zerlegt; an Stelle des Ketols tritt als secundäres Product Bromoform auf, wenn bei gewöhnlicher Temperatur gearbeitet wird. Bei $0^{0}$ scheidet kohlensaures Kalium aus der Lösung der Dibromtetronsäure langsam Tribrommethylketol ab.

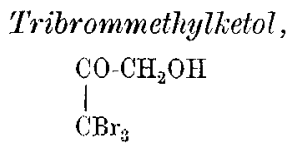

Die Verbindung lässt sich in quantitativer Ausbeute erhalten, wenn man einen Theil Bromtetronsäure, welche in sechs Theilen Wasser suspendirt ist, mit zwei Theilen Brom versetzt und die Flüssigkeit entweder zwei bis drei Tage stehen lässt oder auf dem Wasserbade erwärmt, bis die Kohlensäureentwickelung aufhört; die Lösung soll stets freies Brom enthalten.

Man filtrirt dann von dem ausgeschiedenen Ketol ab, wäscht mit Wasser gut aus und krystallisirt es aus heissem Aceton oder Methylalkohol um.

$\begin{array}{lcr} & \text { Berechnet fiir } & \text { Gefunden } \\ & \mathrm{C}_{3} \mathrm{H}_{3} \mathrm{Br}_{3} \mathrm{O}_{2} & \\ \mathrm{C} & 11,57 & 11,65 \\ \mathrm{H} & 0,96 & 0,90 \\ \mathrm{Br} & 77,17 & 77,49\end{array}$

Die Verbindung löst sich nur wenig in Wasser, Aether, Benzol, Methylalkohol und Aceton, etwas leichter in Aethylalkohol. Aus heissem Aceton oder Methylalkohol krystallisirt. sie in glänzenden, rhombischen Blättchen, die beim Liegen an der Luft nach kurzer Zeit den Glanz verlieren, bei $168^{\circ}$ sintern und bei $174^{0}$ unter lebhafter Gasentwickelung schmelzen. Diesen Schmelzpunkt, oder richtiger Zersetzungspunlit, beobachtet man nur bei Substanz, die sehr oft umkrystallisirt 
worden ist; gewöhnlich schmilzt sie zwischen $150^{\circ}$ und $160^{\circ}$ und lässt dann durch die Analyse Verunreinigungen nicht mehr erkennen. Wir haben lange Zeit geglaubt, dass das rohe, nur mit Wasser gewaschene Product, welches gewöhnlich zwischen $115-125^{0}$ sich zersetzt, ein Gemenge zweier isomerer Verbindungen sei, doch ist es uns nicht gelungen, einen zweiten Körper in reinem Zustande zu isoliren. Fehling'sche Lösung und Permanganat werden von dem Ketol in gelinder Wärme leicht reducirt; warme Sodalösung zersetzt es in Bromoform. Um das dabei entstehende zweite Spaltungsproduct, die Glycolsäure, in einfacher Weise erkennen zu können, wurde die Zersetzung mit Kalkwasser vorgenommen.

\section{Spaltung des Tribrommethylketols in Glycolsäure und Bromoform.}

Das Ketol wurde mit überschüssigem Kalkwasser am absteigenden Kühler gekocht, bis mit den Wasserdämpfen Bromoform nicht mehr überging. Der Destillationsrückstand, mittelst Kohlensäure vom Kalkhydrat befreit und heiss filtrirt, schied beim Erkalten büschelförmig gruppirte Nädelchen aus, welche zur Analyse wiederholt aus heissem Wasser umkrystallisirt wurden. Die bei der Krystallwasserbestimmung gefundenen Werthe stimmen mit den früher ${ }^{11}$ ) erhaltenen überein; es lag glycolsaures Calcium vor.

$0,2464 \mathrm{~g}$ lufttrocknes Salz verloren bei $140^{\circ} \quad 0,0708 \mathrm{~g}$ und gaben $0,125+\mathrm{CaSO}_{4}$.

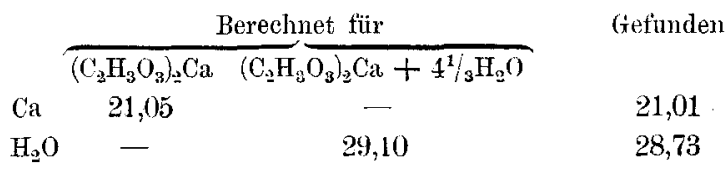

Die aus dem Salz isolirte Glycolsäure schmolz nach dem Umkrystallisiren aus Aether bei $79^{\circ}$.

Das im Destillat enthaltene Bromoform wurde nochmals mit Wasser übergetrieben, mit Chlorealcium entwässert und

\footnotetext{
11) Diese Amalen $\mathbf{2 8 8 ,} 18$. 
destillirt. Der Siedepunkt lag bei $146^{\circ}$. Wir brachten das Oel zum Erstarren, pressten die Krystalle zwischen Filtrirpapier $a b$, entwässerten nochmals und bestimmten wiederum den Siedepunkt. Die gesammte Menge, etwa $6 \mathrm{~g}$, ging dabei ganz constant bei $146^{\circ}$ über ( $751 \mathrm{~mm}$ Druck), erstarrte bei $7,5^{\circ}$ und schmolz in der Capillare bei $9^{0}$. Die folgende Analyse bestätigt, dass die Verbindung in reinem Zustande rorlag.

$0,2022 \mathrm{~g}$ gaben $0,4506 \mathrm{AgBr}$.

$\begin{array}{ccc} & \text { Bereclinet fiir } & \text { Gefinden } \\ \mathrm{CHBr} & & \\ \mathrm{br} & 94,86 & 44,70\end{array}$

Es sei darauf hingewiesen, dass die in der Litteratur sich vortindenden Angaben sowohl über den Schmelzpunkt als auch über den Siedepunkt des Bromoforms zum Theil nicht unerheblich von einander abweichen; der Schmelzpunkt wurde gewöhnlich innerhalb $2-8^{0}$, der Siedepunkt innerhalb $149^{\circ}$ bis $152^{\circ}$ beobachtet. Die obige Bildungsweise bietet an sich scbon eine grössere Garantie für die Reinheit des Materials als die gewöhnliche Darstellungsmethode, und darf man den Schmelzpunkt $9^{0}$ jedenfalls als richtig annehmen. Auch die Angabe über den Siedepunkt $\left(146^{\circ}\right)$ halten wir für zuverlässig, obgleich hierüber wegen der kleinen Quantität, die uns zum Versuche zur Verfügung stand, vielleicht Zweifel lant werden könnten.

\section{Einwirkung von Ammoniak auf llibromtetronsäure.}

Wenn man die wässrige Lösung der Dibromtetronsäure mit concentrirtem Ammoniak rersetzt, so scheiden sich sofort weisse Nadeln $a b$, die nach einmaligem Umkrystallisiren aus heissem Wasser bei $154-155^{\circ}$ schmelzen. Die Terbindung erwies sich identisch mit dem längst bekannten Dibromacetamid.

$0,2240 \mathrm{~g}$ gaben $0,0895 \mathrm{CO}_{2}$ und $0,0277 \mathrm{H}_{2} \mathrm{O}$.

$\begin{array}{ccc} & \text { Berechnet für } & \text { Gefunden } \\ & \mathrm{C}_{\mathbf{2}} \mathrm{H}_{3} \mathrm{Br}_{\mathrm{g}} \mathrm{No} & \\ \mathrm{C} & 11,06 & 10,89 \\ \mathrm{H} & 1,38 & 1,37\end{array}$


Die mit Aether extrahirte Mutterlauge des Amids wurde eingedampft und mit Chlorcalciumlösung zersetzt, wobei das schwerlösliche glycolsaure Calcium in den charakteristischen Formen ausfiel; von einer Analyse honnte Abstand genommen werden.

$$
\begin{gathered}
a-\text { Dibromätlyylketol, } \\
\mathrm{CO}-\mathrm{CH}_{2} \mathrm{OH} \\
\mathrm{CBr}_{2}-\mathrm{CH}_{3}
\end{gathered}
$$

Die so auffallend leicht vor sich gehende Spaltung der Dibromtetronsäure in Tribrommethylketol findet ihr Analogon in dem Uebergange der Brommethyltetronsäure (Bromtetrinsäure) in Dibromäthylketol unter dem Einfluss des Broms. Die Reaction verläuft wahrscheinlich folgendermassen:

$$
\begin{aligned}
& \underset{\mathrm{CH}_{3}-\mathrm{CBr}-\mathrm{CO}}{\mathrm{CO}-\mathrm{CO}}+\mathrm{H}_{2} \mathrm{O}=\stackrel{\mathrm{CO}-\mathrm{CH}_{2} \mathrm{OH}}{\mathrm{CH}_{3}-\mathrm{CHr}-\mathrm{COOH}} \\
& \stackrel{\mathrm{CO}-\mathrm{CH}}{3}-\mathrm{CBr}-\mathrm{COOH}_{\mid}^{\mathrm{COH}}=\overbrace{\mathrm{CBr}_{2}-\mathrm{CH}_{3}}^{\mathrm{O}-\mathrm{CH}_{2} \mathrm{OH}}+\mathrm{CO}_{2}+\mathrm{HBr} .
\end{aligned}
$$

$9 \mathrm{~g}$ Methyltetronsäure wurden in $80 \mathrm{~g}$ Wasser suspendirt und durch $13 \mathrm{~g}$ Brom in Brommethyltetronsäure übergeführt. Die Lösung wurde sodann auf dem Wasserbade erhitzt und so lange mit Brom in kleinen Portionen versetzt, bis die anfangs lebhafte Kohlensäureentwickelung beendet war. Es hatte sich ein Oel abgeschieden, das in Aether aufgenommen, mit Wasser gewaschen wurde und nach dem Verdunsten des Aethers bei mehrtägigem Stehen zum grössten Theile erstarrte. Die auf Thon getrocknete Masse krystallisirte aus einer Mischung von Chloroform und Ligroïn in Form glänzender Blättchen, welche bei $85^{\circ}$ schmolzen und bei der Analyse die für Dibromäthylketol berechneten Werthe gaben.

$0,2071 \mathrm{~g}$, über schwefelsänre getrockuet, gahen $0,145 \tilde{5} \mathrm{CO}_{2}$ and $0,0143 \mathrm{H}_{2} \mathrm{O}$.

$0,1643 \mathrm{~g}$, übcr Schwefelsäure getrocknet, gaben $0,2527 \mathrm{AgBr}$. 


$\begin{array}{lcr} & \text { Berechnet für } & \text { Gefunden } \\ & \mathrm{C}_{4} \mathrm{H}_{65} \mathrm{Br}_{2} \mathrm{O}_{2} & \\ \mathrm{C} & 19,51 & 19,17 \\ \mathrm{H} & 2,44 & 2,27 \\ \mathrm{Br} & 65,04 & 65,43\end{array}$

Die ganz reine, frisch umkrystallisirte Verbindung schmilzt bei $85^{\circ}$, verändert sich aber im Laufe von zwei bis drei Tagen und schmilzt dann um $7-8^{0}$ tiefer. Sie ist leicht löslich in Aether und warmem Chloroform, schwer in Ligroïn und Wasser; Fehling'sche Lösung und ammoniakalische Silberlösung werden durch sie leicht reducirt.

\title{
Verhalten der Tetronsäure und Bromtetronsäure gegen salpetrige Säure.
}

\author{
Oximidotetronsäure,<smiles>O=C1CCCCC1=O</smiles>

Beim Vermischen der Lösungen von Tetronsäure und Natriumnitrit entsteht sofort das Natriumsalz der Oximidotetronsäure, welches der Flüssigkeit eine violette Farbe ertheilt. Zur Gewinnung der wenig beständigen freien Säure kann folgendermassen verfahren werden.

$\mathrm{Zu}$ Tetronsäure, welche mit wenig Wasser übergossen ist, giebt man in Portionen das gleiche Gewicht Natriumnitrit (concentrirte wässrige Lösung), lässt die Flüssigkeit 15 Minuten stehen und säuert mit 12 procentiger Salzsäure unter Eiskühlung langsam an, wobei ein Theil der Oximidotetronsäure in Form gelber Blättchen ausfällt. Man verjagt dann die überschüssige salpetrige Säure, indem man einen Luftstrom durch die Flüssigkeit schickt und schüttelt sehr oft mit Aether aus (bis der Aether eine Sodalösung nur mehr schwach rosa färbt). Bei dem durch Einleiten eines trocknen Luftstromes bewirkten Verdampfen des Aethers bleibt die neue Verbindung als gelbe, krystallinische Masse zurück, welche in Wasser und Alkohol leicht, weniger leicht in Aether, Benzol und Chloroform löslich ist. 
Zur Reinigung nimmt man die Säure in wenig lauwarmem Wasser auf, kühlt die Lösung stark ab und saugt ron den ansgeschiedenen gelben Blättchen rasch ab.

$0,1727 \mathrm{~g}$ gaben $0,2344 \mathrm{CO}_{2}$ and $0,0397 \mathrm{H}_{3} \mathrm{O}$.

$0,1093 \mathrm{~g}, \quad 10,4 \mathrm{ccm}$ Stickgas bei $18^{\circ}$ und $750 \mathrm{~mm}$ Druck.

Berechnet fiir $\mathrm{C}_{4} \mathrm{H}_{3} \mathrm{NO}_{4}$

C $\quad 37,21$

$\mathrm{H} \quad 2,33$

$\mathrm{N} \quad 10,85$
Gefunden

37,01

2,55

10,84

Die Verbindung schmilzt unter Gasentwickelung und unter Bräunung bei $136^{\circ}$, beim raschen Erhitzen des Bades bei $144^{\circ}$. Sie ist eine auffallend starke Säure, welche Carbonate leicht zersetzt unter Bildung von meist violett bis roth gefärbten Salzen. Die Salze der Alkalien, Erdalkalien und des Ammoniaks lösen sich leicht in Wasser mit tief violetter Farbe; aus ihrer Lösung fällt Silbernitrat ein bronzefarbiges, grünlich schillerndes Silbersalz, das sich in Wasser ziemlich leicht mit violetter Farbe löst. In der wässrigen Säurelösung erzeugt Silbernitrat eine violette Färbung, und es scheiden sich bald neben dem grünen Salz hellrothe Aggregate $a b$, die beim Umkrystallisiren aus heissem Wasser in die grünen Formen übergehen.

Bezüglich der stark sauren Eigenschaften, der Farbe der Salze u. s. w. erinnert die Verbindung ausserordentlich an das Oximidomethylsynoxazolon ${ }^{12}$ ), welches ebenfalls ein inneres Anhydrid ist und im Aufbau eine gewisse Aehnlichkeit mit den Lactonen zeigt:

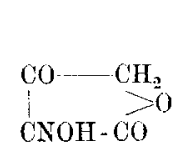

Oximidotetronsäure

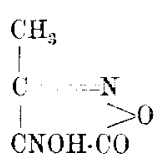

Oxinidomethyloxazolon.

12) Ceresole und Köckert, Ber. d. deutsch. chem. Ges. 17, 823; Nussberger, Ber. d. dentsch. chem. Ges. 25, 2157; Jovitschitsch, Ber. d. deutsch. chem. (ies. 28, 2675. 
Die Oximidotetronsäure ist wenig beständig. Beim Stehen am Lichte wird sie bald braun und schmierig und spaltet beim Kochen mit Wasser Blausäure ab. Ueberschüssige Natronlauge oder Salzsâure zersetzen sie schon bei gewöhnlicher Temperatur. Verdünnte Saipetersäure verwandelt sie in eine schön krystallisirende Säure, welche einfacher durch Nitrirung der Tetronsäure erhalten werden kann. Auch Tetrinsäure lässt sich leicht nitriren. Hierüber soll später berichtet werden.

Auf Bromtetronsäure wirkt salpetrige Säure anders ein als auf Methyltetronsäure (Tetrinsäure). Während letztere Säure unter Aufspaltung des Ringes in Oximidopropioglycolsäure übergeführt wird, entsteht aus Bromtetronsäure die soeben beschriebene Oximidotetronsäure; daneben bildet sich Dibromtetronsäure, herrührend von der Einwirlung der unterbromigen Säure anf einen Theil der Bromtetronsäure. Die Umsetzung verläuft wahrscheinlich in folgender Weise:

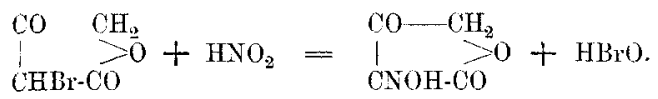

Zu $\tilde{\jmath} g$ fein gepulverter, in wenig Wasser suspendirter Bromtetronsäure wurde unter Kühlung mit Eiswasser eine concentrirte Lösung von $3 \mathrm{~g}$ Natriumnitrit langsam gesetzt. Die Flüssigkeit nahm sofort eine violettrothe Farbe an und schied beim Ansäuem mit 12 procentiger eiskaiter Salzsäure gelbe Blättchen $a b$, die sich nach Schmelzpunkt $\left(143-144^{\circ}\right)$ und sonstigem Verhalten als Oximidotetronsäure zu erkennen gaben. In dem Filtrat dieser Säure konnten Blausäure, Oxalsäure und Dibromtetronsäure leicht machgewiesen werden. Zur Erkennung der letzteren wurde ihr Verhalten gegen Ammoniak benutzt. Auf Zusatz von Ammoniak nahm die Lösung wieder violette Farbe an und schied weisse Nadeln ab, welche bei $154-155^{\circ}$ schmolzen und sich zu Dibromessigsäure verseifon liessen. Die Verbindung war also Dibromacetamid; sie wurde mit folgendem Resuitate analysirt:

$0,1725 \mathrm{~g}$ gaben $9,8 \mathrm{~cm}$ Stickgas bei $20^{\circ}$ und $749 \mathrm{~mm}$ bruck.

$0,1962 \mathrm{~g} \quad$ n $\quad 0,3423 \mathrm{AgBr}$. 


$\begin{array}{lcr} & \text { Berechnet fïr } & \text { Giefunden } \\ & \mathrm{C}_{2} \mathrm{H}_{3} \mathrm{Br}_{3} \mathrm{NO} & \\ \mathrm{N} & 6,45 & 6,40 \\ \mathrm{Br} & 73,73 & 74,24\end{array}$

Es mag hier kurz ein Versuch Erwähnung finden, welcher in der Absicht angestellt wurde, die Bromtetronsäure mittelst Salpetrigsäureanhydrid in die Nitrosobromtetronsäure ${ }^{12}$ ) überzuführen. Die Reaction verlief anders, als nach Analogie mit der Methyltetronsäure zu erwarten war. Das Anhydrid (aus Arsenik und Salpetersäure) wirkte in Eisessiglösung oxydirend auf die Bromtetronsäure ein, führte sie über in Oxalsäure, Tribrommethylketol und vermuthlich Dibromdinitromethan. Das Tribrommethylketol zeigte das früher angegebene Verhalten und gab bei der Analyse folgende Zahlen:

$0,2239 \mathrm{~g}$ gaben $0,0983 \mathrm{CO}_{2}$ und $0,0204 \mathrm{H}_{2} \mathrm{O}$.

$\begin{array}{ccc} & \text { Berechnet fiir } & \text { Gefunden } \\ & \mathrm{C}_{3} \mathrm{H}_{8} \mathrm{Br}_{3} \mathrm{O}_{2} & \\ \mathrm{C} & 11, \tilde{5} 7 & 11,97 \\ \mathrm{H} & 0,96 & 1,01\end{array}$

\section{Dioximidobutyrolacton,}

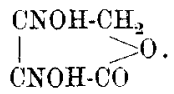

Bei Gelegenheit einiger Versuche zur Gewinnung des Diketobutyrolactons haben wir die Beobachtung gemacht, dass die

12) Eine Bemerkung iiber die Constitution der Nitrosoalkyltetronsäuren (diese Annalen $\mathbf{2 8 8}, 7$ ) möchte ich hier einschalten. Die von Baeyer (Ber. d. dentsch. chem. Ges. 28, 641) ermittelte Thatsache, dass Nitrosocarvon und Nitrosomenthon Bisnitrosoverbindungen sind, welche durch alkoholische Salzsäure eine eigenthümliche Spaltung in Bisnitrosylsäuren erleiden, hat mich die Frage in Erwägung ziehen lassen, ob die Nitrosomethyltetronsäure vielleicht der Reihe der Bisuitrosoverbindungen angehöre. Leider haben diesbezügliche Versuche kein die Frage entscheidendes Resnltat ergeben. Der Mangel an einem geeigneten Lösuugsmittel macht die Molekulargewichtsbestimmung unmöglich; Nitrosoüthyltstronsäure (Schmelzp. 127 $7^{\circ}$ wird ron alkoholischer Salzsäure 
salzsaure Lösung von Oximidotetronsäure bei mehrstündigem Stehen Krystalle von Dioximidobutyrolacton abscheidet. In analoger Weise erhielten Treadwell und Westenberger ${ }^{13}$ ) das Methylglyoxim aus Isonitrosoaceton. Es ist zweifellos, dass in erster Phase das anscheinend nicht sehr beständige Diketobutyrolacton neben Hydroxylamin entsteht, welch letzteres damn mit Oximidotetronsäure das Dioximidobutyrolacton giebt. In der That fällt auch beim Vermischen der wässrigen Lösungen von Oximidotetronsäure und salzsaurem Hydroxylamin das Dioxim in nahezu quantitativer Ausbeute sofort aus.

Bei der Zersetzung der Oximidotetronsäure mittelst verdünnter Salzsäure in der Kälte treten als Nebenproducte Blausäure, Oxalsäure und in kleiner Menge eine schön krystallisirende Säure auf, welche bei etwa $220^{\circ}$ unter Gasentwicklung schmilzt und wahrscheinlich ein Zersetzungsproduct des Dioxims ist.

Das Dioximidobutyrolacton krystallisirt aus heissem Wasser in glänzenden Prismen oder Rhomben - beide oft nebeneinander -, die ein Molekül Wasser enthalten. Das Krystallwasser entweicht langsam in Vacuum, rasch beim Trocknen des Körpers bei $100^{\circ}$.

$0,1646 \mathrm{~g}$, lufttrocken, saben $0,1770 \mathrm{CO} \mathrm{g}_{2}$ und $0,0600 \mathrm{H}_{2} \mathrm{O}$.

$0,1826 \mathrm{~g}, \quad, \quad, 28,2 \mathrm{ccm}$ stickgas bei $23^{\circ}$ und $755 \mathrm{~mm}$ Druck.

$\begin{array}{ccr} & \text { Berechnet für } & \text { Giefiunden } \\ & \mathrm{C}_{4} \mathrm{H}_{1} \mathrm{~N}_{2} \mathrm{O}_{4}+\mathrm{H}_{2} \mathrm{O} & \\ \mathrm{C} & 29,62 & 29,32 \\ \mathrm{H} & 3,70 & 1,05 \\ \mathrm{~N} & 17,28 & 17,28\end{array}$

$0,1596 \mathrm{~g}$, lufttrocken, verloren bei $105^{0} 0,0180$ und gaben $0,1741 \mathrm{CO}_{\mathrm{g}}$ und $0,0397 \mathrm{H}_{2} \mathrm{O}$.

$0,1574 \mathrm{~g}$, lufttrocken, verloren über Schwefelsäure $0,0176 \mathrm{~g}$ und gaben $24,4 \mathrm{~cm}$ Stickgas bei $22^{\circ}$ und $754 \mathrm{~mm}$ Druck.

(bei $0^{\circ}$ gesättigt) kaum angegriffen. Ja num die einfache Formel die einzelnen heactionen \%a erklären im stande ist, so liegt vorläufig kein Grund vor, sie zu verwerfen.

W.

19) Ber. d. deutsch. chem. Ges. 15, 2787. 


\begin{tabular}{lccccc} 
& \multicolumn{2}{c}{ Berechnet für } & & \multicolumn{2}{c}{ Gefunden } \\
\cline { 2 - 4 } $\mathrm{C}_{2} \mathrm{O} \mathrm{H}_{4} \mathrm{~N}_{2} \mathrm{O}_{4}+\mathrm{H}_{2} \mathrm{O}$ & $\mathrm{C}_{4} \mathrm{H}_{4} \mathrm{~N}_{2} \mathrm{O}_{4}$ & & $\overbrace{11,28}$ & 11,18 \\
$\mathrm{C}$ & $\mathbf{1 1 , 1 1}$ & - & & 33,53 & - \\
$\mathrm{H}$ & - & 33,33 & & 3,11 & - \\
$\mathrm{N}$ & - & 2,78 & & 3,61
\end{tabular}

Das Dioxim löst sich sehr leicht in heissem Wasser, ziemlich schwer in Aether, Benzol und kaltem Wasser; es schmilzt in wasserfreiem Zustande unter Gasentwicklung und Bräunung bei $178^{\circ}$. Beim Kochen mit Salzsäure wird es zersetzt. Die wässrige Lösung nimmt anf Zusatz von Eisenchlorid eine rothe, auf Zusatz von essigsaurem Kupfer eine grüne Farbe an; Silbernitrat und essigsaures Blei fällen die Salze in Form gelber, schwer löslicher Niederschläge aus. Die sauren Eigenschaften der Verbindung treten nicht so sehr hervor, wie es bei der Oximidotetronsäure der Fall ist, immerhin vermag das Dioxim noch Carbonate zu zersetzen. Die Krystalle färben sich beim Uebergiessen mit Sodalösung gelb und lösen sich dann langsam auf; beim Verdunsten der gelben Lösung scheidet sich das Natriumsalz in feinen, gelben Nadeln aus. Das Baryumsalz krystallisirt ebenfalls in gelben Nadeln, das etwas schwerer lösliche Kaliumsalz bildet gelbe, glänzende Prismen. Aus den Salzen setzt verdünnte Salzsäure das unveränderte Dioxim in Freiheit. Die gelbe Lösung des Natriumsalzes entfärbt sich auf Zusatz von etwas Natronlange oder beim Kochen mit Soda und enthält dann vermuthlich das Salz der Hydroxysäure, aus dem sich das Dioxim mit Salzsäure nicht wieder abscheiden lässt; wenn man die angesäuerte Lösung kurze Zeit kocht und dann mit kohlensaurem Natrium absättigt, so nimmt sie eine tiefrothe Farbe an, verursacht durch die Bildung des Oxazolons:

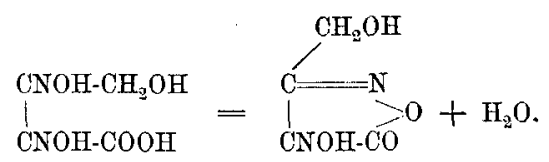

Es liegt in unserer Absicht, diese Reactionen wegen ihres theoretischen Interesses eingehend zu studiren und die ausgesprochene Ansicht zu begründen. 


\section{Oximidobrommethylbutyrolacton, CNOH-CH.$$
\mathrm{CH}_{8}-\mathrm{CBr}-\mathrm{CO}
$$

Fein gepulverte Brommethyltetronsäure (zwei Theile) löst sich in einer kalten, wässrigen Lösung von salzsaurem Hydroxylamin (ein Theil) langsam auf und alsbald beginnt die Abscheidung feiner, glänzender Nadeln der Oximidoverbindung; sie lässt sich am besten aus einer kalten, wasserfreien Mischung von Aether und Ligroïn umkrystallisiren und bildet grosse, wasserhelle Prismen, welche bei etwa $128^{\circ}$ schmelzen.

$0,2200 \mathrm{~g}$ gaben $0,2458 \mathrm{CO}_{2}$ und $0,0622 \mathrm{H}_{2} \mathrm{O}$.

$\begin{array}{ccc}0,2525 \mathrm{~g} & " 16 \mathrm{ccm} \text { Stickgas bei } 17^{\circ} \text { und } 760 \mathrm{~mm} \text { Drnck. } \\ 0,2014 \mathrm{~g} & " \quad 0,1739 \mathrm{AgBr} \text { und } 0,0030 \mathrm{Ag} . \\ & \text { Berechnet für } & \text { Gefunden } \\ \mathrm{C} & \mathrm{C}_{5} \mathrm{H}_{6} \mathrm{BrNO}_{3} & 30,47 \\ \mathrm{H} & 28,84 & 3,14 \\ \mathrm{~N} & 2,88 & 7,35 \\ \mathrm{Br} & 6,73 & 37,83\end{array}$

Weitere Reinigungsversuche batten nicht den gewünschten Erfolg, und wenn somit auch feststeht, dass wir die Verbindung in ganz reinem Zustande nicht in Händen hatten, so kann doch nach ihrem Verhalten und ihrer Bildungsweise kaum ein Zweifel bezüglich der Zusammensetzung walten.

Der Körper hat schwach saure Eigenschaften, färbt sich beim Betupfen mit Soda- oder Ammoniaklösung gelb und geht dann langsam in Lösung.

Die frisch bereitete wässrige Lösung ist gelbgrün gefärbt und wird beim Stehen, rasch auf Zusatz von etwas Salzsäure oder beim Erwärmen farblos.

Kohlensaure Alkalien oder Ammoniak erzeugen in der grünen Lösung schleimige Niederschläge, nicht mehr dagegen in der entfärbten Lösung.

Wasser von $80-90^{\circ}$ zersetzt die Verbindung in Diacetyl, Kohlensänre und bromhaltige Producte, wahrscheinlich im Sinne der Gleichung: 
$\mathrm{CH}_{3}-\mathrm{CBr}-\underset{\mathrm{CO}}{\mathrm{CNOH}-\mathrm{CH}_{2}}+2 \mathrm{H}_{2} \mathrm{O}={\underset{\mathrm{CO}}{\mathrm{COH}}}_{3}^{\mathrm{CO}-\mathrm{CH}_{3}}+\mathrm{CO}_{2}+\mathrm{NH}_{2} \mathrm{OH}-\mathrm{HBr}$.

\section{Anhydrotetronsäure.}

Diese Verbindung bildet sich, wenn man die concentrirte wässrige Lösung der Tetronsäure einige Zeit im Wasserbade erwärmt oder ein bis zwei Wochen bei gewöhnlicher Temperatur stehen lässt. Sie ist in Wasser viel schwerer löslich als die Tetronsäure und scheidet sich aus der Lösung ziemlich vollständig aus.

Aus heissem Wasser krystallisirt die Säure in weissen, zu Wärzchen vereinigten Nädelchen, welche ein Molekül Krystallwasser enthalten.

I. $0,2293 \mathrm{~g}$, lufttrocken, gaben $0,4019 \mathrm{CO}_{2}$ und $0,0797 \mathrm{H}_{2} \mathrm{O}$.

II. $0,2105 \mathrm{~g}, \quad, \quad, \quad 0,3678 \mathrm{CO}_{3} \quad, \quad 0,0735 \mathrm{H}_{2} \mathrm{O}$.

\begin{tabular}{ccrr} 
& Berechnet für & \multicolumn{2}{c}{ Gefunden } \\
& $\mathrm{C}_{8} \mathrm{H}_{6} \mathrm{O}_{5}+\mathrm{H}_{2} \mathrm{O}$ & I. & II. \\
$\mathrm{C}$ & 48,00 & 47,81 & 47,64 \\
$\mathrm{H}$ & 4,00 & 3,87 & 3,88
\end{tabular}

I. $0,2372 \mathrm{~g}$, lufttrocken, verloren über Schwefelsäure $0,0208 \mathrm{~g}$. II. $0,2018 \mathrm{~g}$, $" \quad "$ gaben $0,3547 \mathrm{CO}_{2}$ und $0,0530 \mathrm{H}_{2} \mathrm{O}$.

\begin{tabular}{lcclcc} 
& \multicolumn{2}{c}{ Berechnet für } & & \multicolumn{2}{c}{ Gefunden } \\
\cline { 2 - 5 } $\mathrm{C}$ & $\mathrm{C}_{8} \mathrm{H}_{6} \mathrm{O}_{5}+\mathrm{H}_{2} \mathrm{O}$ & $\mathrm{C}_{8} \mathrm{H}_{6} \mathrm{O}_{5}$ & & & II. \\
$\mathrm{H}$ & - & 52,74 & & - & 52,51 \\
$\mathrm{H}_{2} \mathrm{O}$ & 9,00 & 3,29 & & - & 3,19 \\
& - & - & & 8,77 & 8,72
\end{tabular}

Die Verbindung ist demnach aus der Tetronsäure nach folgender Gleichung entstanden:

$$
2 \mathrm{C}_{4} \mathrm{H}_{4} \mathrm{O}_{3}=\mathrm{C}_{8} \mathrm{H}_{6} \mathrm{O}_{5}+\mathrm{H}_{3} \mathrm{O} \text {. }
$$

Die Anhydrotetronsäure löst sich schwer in Aether, Benzol, Chloroform und kaltem Wasser, ziemlich leicht in Alkohol und heissem Wasser. Sie färbt sich bei $240-245^{\circ}$ gelblich und schmilzt bei $263^{\circ}$ unter vollstänđiger Zersetzung. Die wässrige Lösung wird durch Eisenchlorid roth, durch Natriumnitrit gelb gefärbt; in der sehr concentrirten Lösung ruft Natriumnitrit eine rothbraune Färbung hervor. Sie ist eine starke, einbasische 
Säure, welche Bicarbonate zu zersetzen vermag und durch Kohlensäure aus ihren Salzlösungen nicht ausgefällt wird.

Das Baryumsalz, $\left(\mathrm{C}_{8} \mathrm{H}_{5} \mathrm{O}_{5}\right)_{2} \mathrm{Ba}+5 \mathrm{H}_{2} \mathrm{O}$, durch Absättigen der Säure mit Baryumcarbonat erhalten, krystallisirt in feinen, in Wasser sehr leicht löslichen Nadeln, welche ihr Krystallwasser zum Theil über Schwefelsäure abgeben.

$0,2045 \mathrm{~g}$, lufttrocken, verloren über Schwefelsäure und bei $150^{0}$ $0,0303 \mathrm{~g}$ und gaben $0,0803 \mathrm{BaSO}_{4}$.

\begin{tabular}{|c|c|c|}
\hline \multicolumn{3}{|c|}{$\begin{array}{c}\text { Berechnet für } \\
\left(\mathrm{C}_{8} \mathrm{H}_{5} \mathrm{O}_{5}\right)_{2} \mathrm{Ba}+5 \mathrm{H}_{2} \mathrm{O}\end{array}$} \\
\hline $\mathrm{Ba}$ & 23,26 & 23,10 \\
\hline $\mathrm{H}_{2} \mathrm{O}$ & 15,28 & 14,81 \\
\hline
\end{tabular}

Das Calciumsalz, $\left(\mathrm{C}_{8} \mathrm{H}_{5} \mathrm{O}_{5}\right)_{2} \mathrm{Ca}+5 \mathrm{H}_{2} \mathrm{O}$, mittelst Calciumcarbonat hergestellt, ist in Wasser etwas schwerer löslich als das Baryumsalz und krystallisirt in feinen Nadeln, welche über Schwefelsäure einen Theil ihres Wassers verlieren.

$0,2321 \mathrm{~g}$, lufttrocken, verloren über Schwefelsäure und bei $200^{\circ}$ $0,0422 \mathrm{~g}$ und gaben $0,0639 \mathrm{CaSO}_{4}$.

$\begin{array}{lcr} & \text { Berechnet für } & \text { Gefunden } \\ & \left(\mathrm{C}_{8} \mathrm{H}_{5} \mathrm{O}_{5}\right\rangle_{2} \mathrm{Ca}+5 \mathrm{H}_{2} \mathrm{O} & \\ \mathrm{Ca} & 8,13 & 8,10 \\ \mathrm{H}_{2} \mathrm{O} & 18,29 & 18,18\end{array}$

In der concentrirten wässrigen Lösung erzeugt Silbernitrat einen flockigen Niederschlag, der gegen Licht sehr empfindlich ist und sich in Berührung mit heissem Wasser sofort zersetzt.

Ueber die Constitution der Säure sowie der Condensationsproducte, welche Tetronsäure mit Aldehyden, Ketonen und Ketonsäuren giebt, werden später Mittheilungen gemacht werden.

(Geschlossen am 14. April 1896.) 